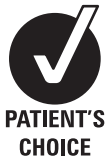

CHOICE

\title{
Concordance between site of onset and limb dominance in amyotrophic lateral sclerosis
}

\author{
M R Turner, ${ }^{1}$ P Wicks, ${ }^{2}$ C A Brownstein, ${ }^{2}$ M P Massagli, ${ }^{2}$ M Toronjo, ${ }^{3} \mathrm{~K}$ Talbot, ${ }^{1}$ \\ A Al-Chalabi ${ }^{3}$
}

${ }^{1}$ Oxford University Department of Clinical Neurology, John Radcliffe Hospital, Oxford, UK ${ }^{2}$ PatientsLikeMe Inc., Cambridge, Massachusetts, USA

${ }^{3} \mathrm{MRC}$ Centre for

Neurodegeneration Research, King's College London, Institute of Psychiatry, London, UK

\section{Correspondence to}

Dr Martin Turner, Department of Clinical Neurology, West Wing Level 3, John Radcliffe Hospital, Oxford OX3 9DU, UK; martin. turner@clneuro.ox.ac.uk

Received 4 February 2010 Revised 6 March 2010 Accepted 12 April 2010 Published Online First 18 June 2010

\section{ABSTRACT}

Background Focality of onset of amyotrophic lateral sclerosis (ALS) is not understood. Attempts to implicate physical exercise in the aetiology of ALS have provided inconsistent results. If physical use of a limb were important in defining the site of onset, then handedness might be expected to influence the side of upper limb-onset disease and footedness likewise in lower limb-onset ALS. Methods ALS patients registered with an internet-based support site were invited to complete an online questionnaire concerning site of onset of symptoms and their dominant hand and foot. A binomial test of proportions was used to investigate the null hypothesis that handedness and footedness do not influence side of onset in upper and lower limb-onset ALS, respectively.

Results 343 ALS patients with limb-onset disease were studied. For upper limb-onset patients, there was concordance for side of onset and handedness (64\%; $\mathrm{p}<0.0006)$. For lower limb-onset patients, concordance for side of onset and footedness was absent. The frequency of left handedness was commensurate with that found in the general population.

Interpretation These results are potentially consistent with the hypothesis that exercise influences pathogenesis in ALS since routine physical demands on the upper limb are heavily influenced by limb dominance, whereas in the lower limbs the commonest function is standing or locomotion, which uses both legs equally. However, there may also be an inherent cortical vulnerability underlying upper limb-onset laterality, possibly influenced by changes in neuronal connectivity and cortical excitability in relation to handedness and reflected by the "split hand" phenomenon consistently observed in ALS.

The site of onset of loss of function in the neurodegenerative disorder amyotrophic lateral sclerosis (ALS) is approximately divided equally between upper limb, lower limb and bulbar territories, ${ }^{1}$ with only a very small proportion $(\sim 3 \%)$ beginning in the respiratory or axial musculature. ${ }^{2}$

Although there are a number of active themes in the quest to understand pathogenesis in ALS, ${ }^{3}$ very little is known about why the onset is focal, though it has been shown that upper (UMN) and lower motor neuron (LMN) signs (the hallmark of ALS) are maximal within this territory and show contiguous spread. ${ }^{4}$

The inconsistent observations of relative athleticism among people with $\mathrm{ALS}^{5-7}$ have led to continued debate about the possible role of physical exercise in the pathogenesis of $\mathrm{ALS}^{8}$ and putative direct effects via excitotoxicity and mitochondrial dysfunction. ${ }^{3}$ A more indirect association, whereby the genetic profiles that underlie ALS susceptibility and athleticism have common components, currently seems equally, if not more, likely.

If physical exercise is a direct determinant of motor neuron damage, then it might be expected that the site onset of symptoms in limb-onset ALS would be biased in favour of the dominant limb through relatively greater use. We attempted to confirm the null hypothesis that the side of initial symptoms in limbonset ALS is unrelated to limb dominance.

\section{METHODS}

Patientslikeme.com is an internet-based information and support resource for patients with a variety of diseases. ALS patients who had selfregistered on the site were invited to complete an anonymous on-line questionnaire to establish the site of onset of their symptoms and their dominance for upper limb (UL) use (handedness) and lower limb (LL) use (footedness). The specific hypothesis was not mentioned to reduce bias, and respondents were only able to submit one questionnaire.

Independence of variables was tested using $\chi^{2}$ for categorical variables and linear regression for continuous variables.

A binomial test of proportions was used to establish the degree of concordance between the side of onset and handedness or footedness for upper and lower limb-onset ALS patients, respectively. Under the null hypothesis, side of onset and limb dominance should be independent and, therefore, equally likely.

All calculations were performed using SPSS (V.14, SPSS Inc).

\section{RESULTS}

\section{Overall group}

A total of 502 patients responded (estimated to be $75 \%$ of the total possible). Seventy-two patients were either unable to specify the site of symptom onset $(n=13)$ or entered more than one site for initial symptoms $(n=59)$ and were removed from the subsequent analysis. Of the remaining 430 patients, $55 \%$ were male. The mean age of respondents was 56 years (SD 10, 26-82); there were no significant differences in age with respect to sex. The site of initial symptoms was bulbar in 18\%, UL $37 \%$ (right 23\%, left 14\%), LL 43\% (right 24\%, left 19\%) and respiratory $2 \%$. There was no significant difference in the proportion of left-handedness or leftfootedness for males versus females, nor for bulbar versus limb-onset patients. The male:female ratio was significantly reversed for bulbar (0.7:1) versus limb-onset patients $(1.3: 1 ; p=0.006)$ but with no significant difference in mean age. 


\section{Limb-onset sub-group}

A total of 343 limb-onset patients were available for analysis. For handedness, $87 \%$ reported right dominance, 10\% left and 3\% equal. For footedness, $86 \%$ reported right dominance, $10 \%$ left and $4 \%$ equal. Ten patients (3\%) reported discordance for upper and lower limb dominance.

\section{Concordance of handedness to side of UL onset}

Excluding those reporting equal dominance for handedness $(n=11)$, for upper limb-onset patients, there was concordance for side of onset and handedness: 97 of 151 (64\%) overall (right 90 of 139 and left 7 of 12); $p<0.0006$.

\section{Concordance of footedness to side of LL onset}

Excluding those reporting equal dominance for footedness $(n=13)$, for the lower limb-onset patients, concordance for side of onset and footedness was absent: 99 of 181 (55\%) overall (right 91 of 162 and left 8 of 19); $p=0.234$.

\section{DISCUSSION}

This study demonstrated high concordance for handedness and side of upper limb-onset ALS but no similar relationship in relation to footedness and side of lower limb onset.

For the upper limb-onset patients, our finding is potentially consistent with the hypothesis that exercise influences pathogenesis in ALS, since routine physical demands on the upper limb are heavily influenced by limb dominance. For the lower limb-onset patients, the lack of an effect of footedness is also consistent with this hypothesis since the commonest function is standing or locomotion, which uses both legs equally.

However, another potential reason for a difference in the result between upper and lower limb onset is some inherent vulnerability in upper limb motor neuronal organisation and laterality. There is evidence for greater connectivity in the dominant motor cortex with respect to handedness ${ }^{10}$; specifically, it has been noted that the production of movements in dominant and non-dominant hands involves distinct cerebral networks. ${ }^{11}$ Evolutionary aspects of neocortical function might have relevance to ALS pathogenesis, in that humans have evolved uniquely complex levels of corticomotoneuronal organisation and connectivity as part of the switch to bipedal locomotion and particularly the development of fine hand control through an opposable thumb. ${ }^{12}$ Such complexity may have come at a price and harbour a vulnerability in the ageing motor system unmasked by ALS. The demonstration of a cortical correlate of the "split hand" phenomenon (preferential wasting of the lateral thenar aspect of the hand) in ALS patients ${ }^{13}$ suggests that it is at least plausible that upper limb-onset ALS favours the dominant hand through increased cortical connectivity. Furthermore, in light of the demonstration of cortical hyperexcitability as a feature of early sporadic ALS ${ }^{14}$ and as a preceding event in familial ALS, ${ }^{15}$ it is noteworthy that a transcranial magnetic stimulation study in relation to handedness in healthy participants demonstrated a shorter cortical silent period (reflecting reduced inhibitory function) in the dominant hand. ${ }^{16}$

A study of 21 cases of monomelic atrophy (a purely LMN syndrome) showed no relationship to handedness. ${ }^{17}$ Although not ascertained in our study, it may be pertinent to explore handedness in relation to upper limb-onset ALS further with respect to the degree of UMN involvement clinically.

Finally, with reference to the proposal that ALS may be associated with previous athleticism, although left handedness has been noted to be higher among the sporting elite, reaching $50 \%$ in some disciplines, ${ }^{18}$ we did not observe any increase in left handedness in ALS patients compared with the general population.

\section{Limitations}

The use of a questionnaire-based survey is potentially subject to responder and recall bias. In respect of responder bias, however, the demographics, the distribution of the frequencies of the sites of symptom onset and the sex ratio differences between bulbar and limb onset suggest that our responders are representative of the general ALS population. With respect to recall bias, it is likely that weakness will be noticed sooner in a dominant limb if both limbs are equally weak because of the greater awareness and higher use. While this cannot be completely excluded as an explanation for our findings, it would not be consistent with our overall observation of focal onset of disease. There is also a potential discrepancy between questionnaire and performancebased measures of footedness that may have confounded the results in relation to this. ${ }^{19}$

Prospective, clinic-based studies of handedness in relation to upper limb-onset ALS are needed to confirm our findings, and further research aimed specifically at understanding more about corticomotoneuronal connectivity might be revealing.

Acknowledgements We thank the Medical Research Council, Motor Neurone Disease Association of Great Britain and Ireland, the NIHR for Biomedical Research and those patients registered with http://Patientslikeme.com who took the time to return the questionnaire.

Funding MRT is supported by the MRC/MNDA Lady Edith Wolfson Clinician Scientist Fellowship. PW, CAB, and MPM are employees of PatientsLikeMe and own stock options in the company. PatientsLikeMe receives research support from UCB, Novartis, and Avanir.

\section{Competing interests None.}

Provenance and peer review Not commissioned; externally peer reviewed.

\section{REFERENCES}

1. Haverkamp LJ, Appel V, Appel SH. Natural history of amyotrophic lateral sclerosis in a database population. Validation of a scoring system and a model for survival prediction. Brain 1995;118(Pt 3):707-19.

2. Shoesmith CL, Findlater K, Rowe A, et al. Prognosis of amyotrophic lateral sclerosis with respiratory onset. J Neurol Neurosurg Psychiatry 2007:78:629-31.

3. Rothstein JD. Current hypotheses for the underlying biology of amyotrophic lateral sclerosis. Ann Neurol 2009;65(Suppl 1):S3-9.

4. Ravits J, Paul P, Jorg C. Focality of upper and lower motor neuron degeneration at the clinical onset of ALS. Neurology 2007;68:1571-5.

5. Felmus MT, Patten BM, Swanke L. Antecedent events in amyotrophic lateral sclerosis. Neurology 1976;26:167-72.

6. Kurtzke JF, Beebe GW. Epidemiology of amyotrophic lateral sclerosis: 1. A casecontrol comparison based on ALS deaths. Neurology 1980;30:453-62.

7. Chio A, Benzi G, Dossena M, et al. Severely increased risk of amyotrophic lateral sclerosis among Italian professional football players. Brain 2005;128:472-6.

8. Harwood CA, McDermott CJ, Shaw PJ. Physical activity as an exogenous risk factor in motor neuron disease (MND): a review of the evidence. Amyotroph Lateral Scler 2009;10:191-204

9. Scarmeas $\mathbf{N}$, Shih T, Stern $Y$, et al. Premorbid weight, body mass, and varsity athletics in ALS. Neurology 2002:59:773-5.

10. Hammond G. Correlates of human handedness in primary motor cortex: a review and hypothesis. Neurosci Biobehav Rev 2002:26:285-92.

11. Walsh RR, Small SL, Chen EE, et al. Network activation during bimanual movements in humans. Neuroimage 2008:43:540-53.

12. Eisen A. Amyotrophic lateral sclerosis-evolutionary and other perspectives. Muscle Nerve 2009; 40:297-304.

13. Weber M, Eisen A, Stewart $H$, et al. The split hand in ALS has a cortical basis J Neurol Sci 2000;180:66-70.

14. Prout AJ, Eisen AA. The cortical silent period and amyotrophic lateral sclerosis. Muscle Nerve 1994;17:217-23.

15. Vucic S, Nicholson GA, Kiernan MC. Cortical hyperexcitability may precede the onset of familial amyotrophic lateral sclerosis. Brain 2008;131:1540-50.

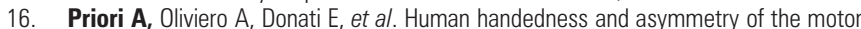
cortical silent period. Exp Brain Res 1999;128:390-6.

17. Gourie-Devi M, Gururaj G, Vasisth $\mathrm{S}$, et al. Risk factors in monomelic amyotrophy: a case control study. NIMHANS J 1993;11:79-87.

18. Raymond $\mathbf{M}$, Pontier $\mathrm{D}$, Dufour $A B$, et al. Frequency-dependent maintenance of left handedness in humans. Proc Biol Sci 1996:263:1627-33.

19. Grouios G, Hatzitaki V, Kollias N, et al. Investigating the stabilising and mobilising features of footedness. Laterality 2009;14:362-80. 